\title{
Review of: "Atom-Precise Fluorescent Copper Cluster for Tumor Microenvironment Targeting and Transient Chemodynamic Cancer Therapy"
}

\author{
Zhao Xueli $^{1}$ \\ 1 Zhengzhou University
}

Potential competing interests: The author(s) declared that no potential competing interests exist.

This paper reported an atomically precise nanocluster Cu6(C4H3N2S)6 (Cu6NC), which could slowly break and generate ROS once encountered with acidic. The stability of material and effect for therapy was mutually contradictory. And the work is not investigated deeply. I don't think it could be published in this journal.

1. The author used 'Tumor microenvironment targeting' in the title, when we say targeting, there is a target (such as folic acid receptor, ). Only pH responsive can not be targeting.

2. Tumor microenvironment exhibited extracellular $\mathrm{pH}$ at 6.5 but intracellular $\mathrm{pH}$ at 7.4. The author demonstrated no ROS production at neutral $\mathrm{pH}$ and ROS production at $\mathrm{pH}$. So it could not be understand the cytotoxicity difference between normal cell and tumor cell in vitro.

3. There is no Ligand and copper complex group as control to verify the effect of copper cluster. The obtained results by now could not demonstrat their statements.

4. The crystal structure could be destroyed by hcl. The state and its change of copper cluster in cell culture medium and intracellular was not investigated. Such as the size distribution and structure stalibity. 